\title{
Environmental Science in Building (6th edition)
}

\author{
Randall McMullan
}

Palgrave Macmillan, Basingstoke, UK; 2007: ISBN 978-02305-25368; 400pp; $£ 32.99$; paperback

Journal of Building Appraisal (2008) 4, 141-142. doi:10.1057/jba.2008.31

This book is intended as a general text on environmental science for students of various disciplines within the built environment, including architecture, construction and surveying. It focuses broadly on science, technology and services and would be suitable as an introductory text/primer for students studying building services engineering who may thereafter require a more in-depth examination of many of the topics.

This is the latest edition of a long published textbook and is presented well with clear diagrams and supplementary materials available easily via a website (http://www. palgrave.com/engineering/builtenvironment/McMullan/index.html). The new edition has been reorganised with a separate 'resource' section, the intention of which is to give a better 'flow' for the primary topics. The balance between topics that are 'primary' and those that are 'principles' has perhaps been a difficult one to achieve at a first attempt. The chapter on Electricity Supplies still has 'Electrical Principles' within it rather than relegating these to the resource section. Similarly the chapter on Aspects of Sound might best be relegated in its entirety to the resource section in future editions of the book - at present the nature of sound is deemed a resource, whereas the nature of hearing is not and this seems inconsistent when the aims of the book are on science in buildings.

Although the information provided in the various chapters is exhaustive, the ordering and the logic of some of the chapters in relation to one another are unclear. As an example, the chapter on 'Thermal Effects in Buildings' discusses thermal insulation in an in-depth and logical manner. A more general chapter follows this on 'Energy Use' with, of necessity, a section on heat losses and the factors affecting heat losses, such as insulation. It would perhaps have been more logical to move from the general to the specific in such a case and reverse the order of these chapters. A similar argument could be made for the lighting chapters with the properties of lighting being followed by artificial lighting, followed by natural lighting.

The contents of some of the chapters are unexpected. 'Air Control in Buildings' includes a discussion on Ground Source Heat Pumps and their efficiencies and also a section on condensation risk assessment. As the book deliberately does not cover building services installations such as heating in any depth, there is little evidence or information on which to appraise or compare with that provided on heat pumps.

The chapter on Green Buildings sits uncomfortably in the book and implies that these are something special or novel. Identifying 'future buildings' in a separate section and consideration of the driving rain index and other aspects of the climate here further compounds the issue. Some, if not all of this information would be more appropriately located in the opening chapter of the book. This is a difficult area to address and discuss given the rapidly changing nature of construction and property but such issues and solutions to design challenges are examples of good current practice. The introduction of 
Energy Performance Certificates, changes to Building Regulation requirements, the Code for Sustainable Homes, the renewables debate and the rising costs of fuels, to name just a few, all make the issue mainstream rather than bolt on.

Overall the book provides a good introductory text to the subject and is of sufficient depth to suit many disciplines. It could be improved by more attention to the division between principles and primary topics and a more logical arrangement of chapters based upon the principles of environmentally sound building design.

Graham Capper

Programme Leader for Building Surveying, School of the Built Environment, Northumbria University, UK 